\title{
Measurement and Analysis of the Positron Annihilation Lifetime Spectra for Mesoporous Silica
}

\author{
R. ZALESKI* \\ Institute of Physics, Maria Curie-Skłodowska University \\ pl. Marii Curie-Skłodowskiej 1, 20-031 Lublin, Poland
}

Positron annihilation lifetime spectroscopy as a technique of porous materials investigation is still under development. Both measurement conditions and lifetime spectra analysis methods have to be adapted to the requirements of positron porosimetry. If complex positron annihilation lifetime spectra of porous materials are processed, numerical methods of lifetime spectra analysis have to be used with a particular care. Among various methods of positron annihilation lifetime spectra analysis available, the most popular ones were tested implemented in programs MELT and LT. It was found, from the simulated spectra study, that a large number of counts in a spectrum is needed to obtain reliable results of analysis. Even then only an approximate solution is available. Determination of the approximation nature helps in a further interpretation of the results and in positron annihilation lifetime spectrometer setting adequate to the problem. For this purpose, a few complex spectra obtained from positron beam facility were tested. The results show a negligible influence of resolution function on long lifetime components, while the precise estimation of the background level is crucial. Also some distortions caused by imperfection of numerical analysis methods are pointed out.

PACS numbers: 78.70.Bj, 36.10.Dr, 61.43.Gt, 81.07.--b, 41.75.Fr

\section{Introduction}

An amount of information which can be obtained from positron annihilation lifetime (PAL) spectra depends on many factors. First of all, the quality of the spectra depends on experimental conditions, i.e. PAL spectrometer configuration and settings. The important phase of investigation using PAL technique is a numerical analysis of the spectra. A few methods are accessible, like fitting a simplified model function $[1,2]$ or deconvolution $[3,4]$. The results depend highly on

*e-mail: radek.zaleski@umcs.lublin.pl 
assumptions made during the analysis. Even so, the exact solution of the problem is not obtainable; however, even approximate results provide a valuable piece of information. In order to interpret them correctly, their possible distortions have to be known. A variety of materials is investigated by positron annihilation lifetime spectroscopy and a wide range of lifetimes is observed. If only short (i.e. of the order of hundreds picoseconds) mean lifetimes are present, problems, encountered during measurement and spectra processing, differ from those appearing in the case when positronium lifetimes are close to the vacuum value of $142 \mathrm{~ns}$. It concerns, e.g. porous insulators, where the components with the lifetimes from $100 \mathrm{ps}$ to more than $100 \mathrm{~ns}$ are present. A correct separation of all short and long spectrum components would be difficult if not impossible. Fortunately, the most interesting information about properties of porous materials can be obtained from lifetimes and intensities of ortho-positronium components, which have lifetimes of the order of tens nanoseconds. Thus some simplifications causing distortions of short-lived components are acceptable if they help in correct revealing of long-lived ones. What has to be known is whether the incorrect results are due to inaccurate assumptions made during analysis, or to the presence of spectra distortions arisen during measurement. If this requirement is fulfilled, the corrections to spectra or analysis conditions can be made or, if this is impossible, the known distortions may be taken into account in the interpretation of the results. In an extreme case, one may found that positron annihilation lifetime spectroscopy cannot be applied to the investigated problem at the present state of PAL technique development. This work presents the study of application of positron annihilation lifetime spectroscopy for a wide class of mesoporous silica materials.

\section{Methods of numerical analysis}

Usually PAL spectra for mesoporous silica consist of several ortho-positronium components that often have the distribution of lifetimes reflecting the different sizes of pores and their size distribution [5]. However, by focusing on long lifetimes it is possible to extract from the spectra more information than it is usually done for short lifetimes. The components having intensity less than $1 \%$ can be separated and shape of usually present distribution of lifetimes can be reconstructed. In order to achieve such results, relative contribution of statistical dispersion should be minimized, so a large number of counts in the spectrum is required. The sufficient number of counts depends on a chosen method of numerical analysis. In this work three most popular methods were applied to the spectra analysis:

1. Fitting the model function (MF) composed of a sum of exponential components $Z(t)$ convoluted with resolution function $R(t)$ approximated by a sum of Gauss functions

$$
N(t)=N_{0} \int_{0}^{\infty} R\left(t-t^{\prime}\right) Z\left(t^{\prime}\right) \mathrm{d} t^{\prime}+T,
$$




$$
Z(t)=\sum_{i=1}^{n} \frac{I_{i}}{\tau_{i}} \exp \left(-\frac{t}{\tau_{i}}\right),
$$

where $N_{0}$ - the total number of counts, $T$ - the background of random coincidences, $I_{i}$ - the $i$ th component's intensity, $\tau_{i}$ - the $i$ th component's lifetime. This method was used by the first numerical routines (like POSITRONFIT [1]) dedicated for the analysis of positron lifetime spectra. It is still very popular because it involves as little fitting parameters as possible, giving the most consistent results and keeping low requirements for hardware. However, using MF method for analysing the spectra of porous materials one has to remember that approximation of lifetime distribution by $\delta$ function is usually inaccurate. Efficient fitting routine and convenient interface for MF is provided by LT programme [2], and that one was used for a further analysis.

2. Fitting of model function where some of $\delta$ lifetime components are replaced by lognormal distributions of lifetimes (DMF). Even being limited to lognormal shape only, the modified model function gives a much better approximation of lifetime spectrum. Implemented in LT programme the DMF model works fine if only one dispersed component is present, giving additional information about width of the distribution. There is also a possibility to evaluate component's lifetime distribution substituting it by a sum of two exponential components [6-8].

3. Deconvolution using Bayesian methods with maximum entropy principle as regularization factor. In that case, there are no assumptions about a number of components - lifetime grid is used and the result is a set of intensities calculated for each point of the grid. Disadvantageous feature of deconvolution is that many parameters like resolution function and zero of time scale have to be known a priori. Deconvolution was adapted for positron lifetime spectra analysis in MELT routine [4]. There is also a method of deconvolution by numerical Laplace inversion (CONTIN program [3]), but it was found that results of MELT are more accurate [9]. Applying MELT to typical lifetime spectra where a lifetime spectrum is registered in thousands of channels, demands quite large routine's requirements for computer memory (about 2 GB for a spectrum in 7000 channels) and CPU speed (a few minutes on $3.2 \mathrm{GHz}$ CPU for the same spectrum).

\section{Number of total counts in PAL spectra}

In order to test the abilities of each of these methods and estimate the number of counts sufficient to obtain accurate results, a set of spectra with various numbers of total counts $\left(10^{6}-10^{8}\right)$ was simulated. Histogram of lifetimes (Fig. 1) obtained by MELT (exponential lifetime grid made of 300 points ranged from 50 ps to $200 \mathrm{~ns})$ as a result of analysis of MCM-41 [10] spectrum was used as the lifetime 

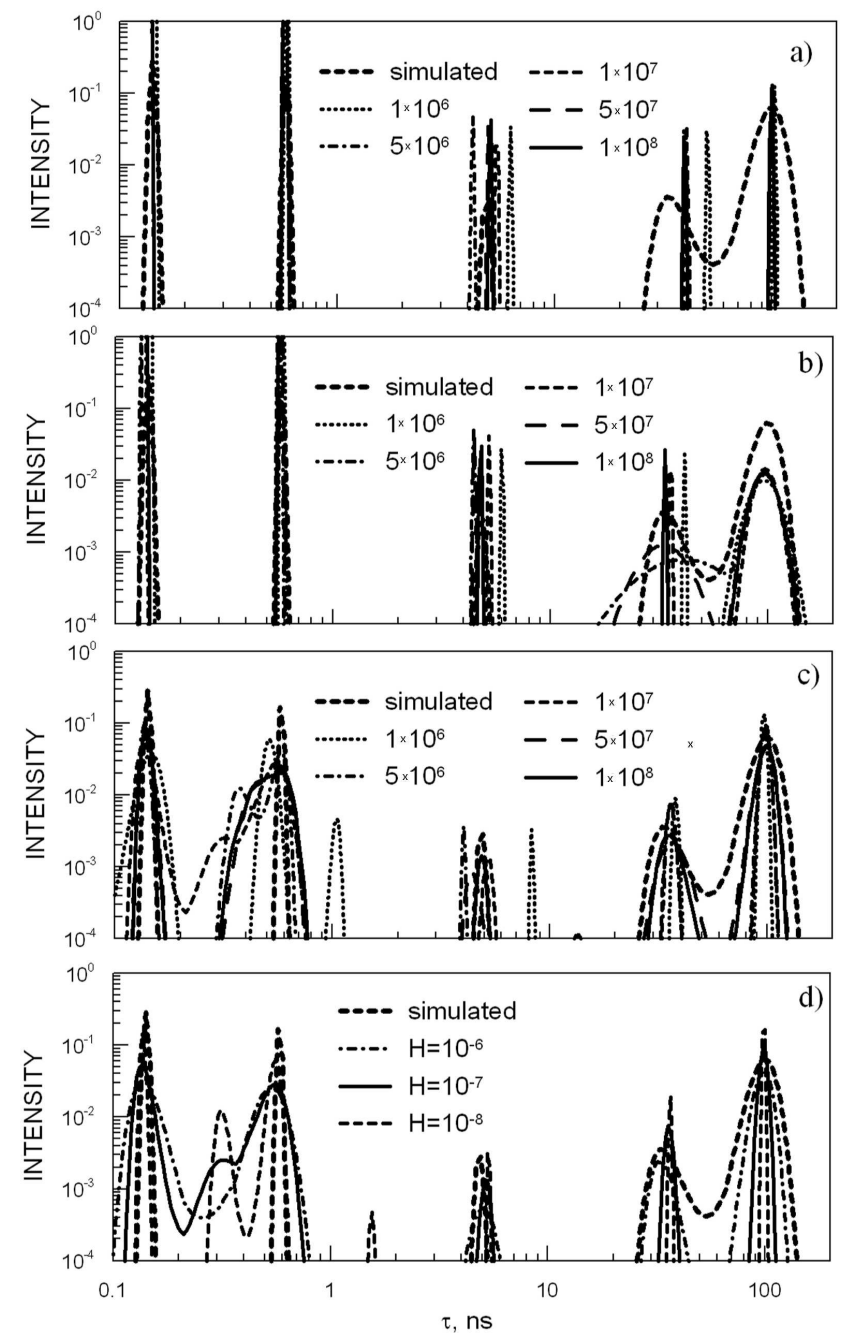

Fig. 1. (a) $\delta$ function components fitted by LT. (b) $\delta$ function components and lognormal lifetime distributions. (c) Histogram of lifetimes obtained by MELT with maximum entropy set to $H=10^{-7}$. Analysed spectra were simulated assuming lifetime distribution as shown by thick dashed line. A total number of counts in the spectra was $10^{6}-10^{8}$ as described in figures. $\delta$ type distributions are presented above as having lognormal distribution with standard deviation $1 \%$ in order to compare their intensities to the components with dispersion of lifetimes.

distribution in simulated spectra. The resolution function was assumed to be a sum of three Gauss functions obtained from LT analysis of a high purity silicon lifetime spectrum. The background of random coincidences was chosen to be $15.5 \%$ of total count number as in the MCM-41 spectrum. 
The results of analysis of some simulated spectra using three described above methods are presented in Fig. 1. In analysis, the resolution function was approximated by two Gauss functions, so $0.5 \%$ contribution of simulated third Gauss function served as unknown (in real spectra) deviation of resolution function from the assumed shape. The MF method (Fig. 1a) reveals narrow shortest components very well. Problems appear for the low-intensity $(I<1 \%)$ third component. Its lifetime is estimated with only $40 \%$ accuracy for less than $10^{7}$ total counts in the spectra. Fitted lifetime $\left(\tau_{4} \approx 39.4 \mathrm{~ns}\right)$ of the fourth component (a few percent intensity) is about $14 \%$ longer than in maximum of the simulated distribution and $11 \%$ longer than the weighted average over the distribution. Only the lifetime for spectrum with $10^{6}$ counts shows a distinct deviation from this value. The longest and most intense component is fitted with a high accuracy.

An attempt to reveal the information about width of two longest lifetimes by assuming their lognormal distributions was made (Fig. 1b). The fitting method DMF shows a similar accuracy as the MF in the case of $\delta$-like short components. Fitted distributions of lifetimes can be divided into two groups for the sake of correctness of $\tau_{4}$ distribution reconstruction. The first group of spectra shows more or less accurate wide distributions (e.g. $5 \times 10^{6}$ and $5 \times 10^{7}$ total counts in Fig. 1b) while in the rest of spectra the fourth component was found to have no meaningful dispersion of lifetimes. Such a misfit occurs even for spectrum with a total number of counts of $10^{8}$.

Finally, MELT was used for the spectra deconvolution. The values of parameters such as resolution function and time zero were assumed as based on LT results of fitting a sum of two Gauss functions. It was found that the maximum entropy parameter influences mostly the width of distributions with a little shift of its maximum to longer lifetimes for narrow distributions (Fig. 1d). An artificial component with a lifetime of $400 \mathrm{ps}$ and an intensity of $0.2 \%$ appears for $H<10^{-7}$. It is the result of not accurate fitting of the resolution function and zero of time scale by LT. This effect disappears if exact simulated values are used in deconvolution. The obtained dispersions are still wider than the simulated ones. The results obtained with the maximum entropy parameter set to $H=10^{-7}$ for each of spectra with various numbers of counts are presented in Fig. 1c. The lifetime histograms differ distinctly. Fortunately, in the most interesting range of three longest components changes are smaller than in the case of the short ones. Third component's lifetime has a correct value if the number of total counts exceeds $10^{7}$. Also dispersions of the two longest components is close to the simulated one (but still narrower) for the spectra containing over $10^{7}$ counts.

\section{Measurement of long lifetimes}

The results described above show that in order to perform the spectra analysis correctly the total number of counts in the spectrum should not fall below $10^{7}$. So, the high rate of counts is needed to get a reasonable measurement time. It 
can be achieved by proper setup of PAL spectrometer. First of all, an intense (e.g. $0.5 \mathrm{MBq}$ ) positron source and high efficiency scintillation crystals (like $\mathrm{BaF}_{2}$ ) should be used. Summing and backscattering effects in these crystals can be a source of distortions. To avoid them, a proper geometry of measurement (e.g. sample moved outside the slit between counters) should be set [11]. In a PAL spectrometer, the parameter that mostly influences counting rate, is energy windows setup. It should be noticed that a meaningful fraction of ortho-positronium annihilates with an emission of three $\gamma$ quanta if its mean lifetime in a sample is of the order of hundred nanoseconds. The energy spectrum in this case is continuous from zero to $511 \mathrm{MeV}$. If STOP energy window does not cover this range, intensities of the longest components in the spectrum are suppressed. A wide (e.g. 150-600 MeV) STOP energy window is beneficial if one wants to obtain a high rate of counts. Due to different detector efficiency for STOP (0-511 keV) and START $(1274 \mathrm{keV})$ quanta detection, the count rate in a lifetime spectrum is limited mostly by the efficiency of START radiation detection. Setting the lower limit of START energy window below the Compton edge increases significantly the count rate. Unfortunately, high activity of the source and wide energy windows deteriorate the two main factors distorting lifetime components - spectrometer resolution function and background of random coincidences.

The more complex resolution function - the less accurate is its approximation during the analysis. Positron beam based measurement conditions result in a much more complex resolution function than in the case of ${ }^{22} \mathrm{Na}$ source. The influence of resolution misfit on the parameters of lifetime components for porous methyl silsesquioxane (MSSQ) positron lifetime spectra [12] was studied. The spectra were provided by the Department of Physics at the Washington State University. In order to simplify a further spectra analysis, the shape of the resolution function should be well known. The lifetime spectrum of positrons in high purity graphite $\left(5 \times 10^{7}\right.$ total counts $)$ proved that a sum of six Gauss functions is needed to approximate the resolution function. The sum can be reduced to three functions, but an artificial component $(\tau \sim 400 \mathrm{ps})$ is needed to get a proper fit. If a porous material is investigated, such component distorts the intensity and lifetime of free positron component. The MSSQ spectra were analysed using the three methods described above. It was found that various combinations of the resolution function and two shortest components parameters lead to a comparable good fit. But even if the two shortest lifetimes change in the range of tens percent there is no significant difference in lifetimes and intensities of the longest components.

Quite different is the influence of the background level on numerical analysis results. First of all, the signal to background ratio decreases if wide energy windows are set. It may result in covering the long lifetime part of the spectrum by statistical dispersion. For example, in the MSSQ spectra (background to total counts: $10.3 \%$ ) a number of counts in the channel originating from the longest component exceeds statistical error only if positron lifetimes are shorter than 500 ns. Another 
source of distortion is a possible non-linearity of spectrometer. It is getting worse if a PAL spectrometer is highly loaded as recommended above. The methods of PAL spectra analysis assume a flat background. The linearity distortion would result in incorrect estimation of its level. To estimate possible effects of such distortion, the same MSSQ spectra as in the case of resolution were analysed assuming various background levels. The results show the importance of this parameter - even $1 \%$ deviation of a background level causes a few percent changes of long components lifetimes and intensities if the MF method is used. When lifetime dispersion is accepted, the results are even worse. It is interesting that the results obtained by MELT depends on the background level in a quite different way than in the case of LT (Fig. 2). MELT finds that both lifetime and its dispersion decrease if the background rises. Also dispersions obtained by LT are decreasing with the background rise, but the lifetime values increase until the dispersion of the longest component is close to zero. Then both lifetimes start to decrease like in the case of MELT.
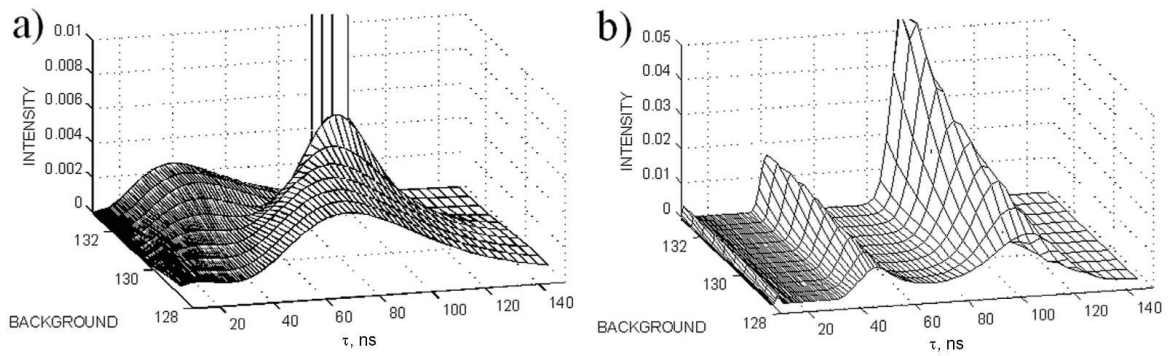

Fig. 2. Change of $o$-Ps lifetime distribution as a function of assumed background value obtained by LT (a) and MELT (b) for the same spectrum.

The results presented lead to the conclusion that one should focus on getting good spectrometer linearity, while good resolution is not necessary if only long lifetimes are object of interest.

\section{Remarks on MELT}

Originally MELT was designed and tested to perform analysis which results in lifetimes of a few nanosecond. Deconvolution of spectra that give the lifetimes a few times longer, as observed in porous silica, often present difficulties. An early assumption that a single Gauss function is sufficient to approximate the resolution function cannot be accepted in the most cases - calculations do not converge to solution. Also the routine determining zero of time scale fails if zero is shifted far from the spectrum maximum. Fortunately, MELT version 4.0 [13] allows one to avoid these problems - time zero fixing and assuming resolution as a sum of Gauss functions were introduced in this version. Still there is one unsolved problem making MELT results questionable. MELT results are a set of 


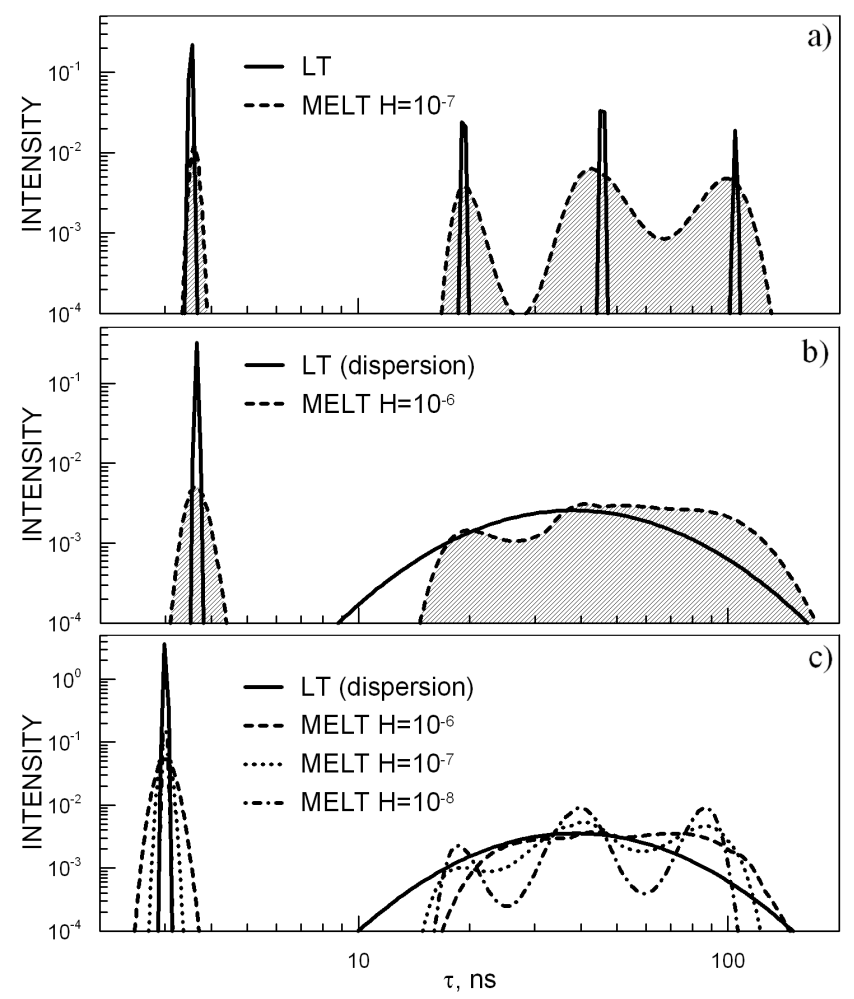

Fig. 3. Simulated spectrum with a wide dispersion of lifetimes. Dashed line represents lifetime histograms obtained by MELT at a maximum entropy parameter value of $H=$ $10^{-7}$ (a) and $10^{-6}$ (b) for the MSSQ spectrum. (c) MELT results for three various $H$ values are shown for the simulated spectrum with a wide dispersion of lifetimes. Solid line shows LT results assuming $\delta$ function components only (a), or lognormal distribution of lifetimes (b and c). $\delta$ type distributions are presented as having lognormal distribution with a standard deviation $1 \%$ in order to compare their intensities to components with dispersion of lifetimes.

solutions depending on a maximum entropy value. These solutions often lead to a comparable fit accuracy. Interpreting the results, one of the solutions has to be chosen based on other premises. For example, MSSQ spectrum deconvolution results for maximum entropy $10^{-7}$ and $10^{-6}$ are presented (Fig. 3). Two different interpretations seem to be possible in the case of lifetimes longer than 10 ns: three narrow lifetime components or one wide distribution of lifetimes. Model functions representing these assumptions were fitted to the spectra by LT. The results were shown in the same figures in comparison. However, it is known that MELT has a tendency to split a wide distribution of lifetimes into two narrower ones for polymers $[6,9]$. In order to test this effect in the range of long lifetimes the spectrum was simulated assuming a wide lognormal dispersion of lifetimes. A set 
of results for various maximum entropy values was obtained by MELT (Fig. 3c). It is clearly seen that for a low maximum entropy the lifetime distribution is separated into a few components while LT with correct assumptions results in as-simulated values. This fact implies that in many cases MELT does not allow one to determine unequivocally the real shape of lifetime distribution. Additional criteria, like results of other porosimetric methods, have to be used.

\section{Conclusions}

Over $10^{7}$ total counts collected in the spectrum assure reliable results of fitting by LT $\delta$-like lifetime spectrum components and deconvolution by MELT. If more than one component with lognormal dispersion of lifetimes is fitted, there is no certainty of correctness of the results even if the total number of counts exceeds $10^{8}$.

A high activity positron source and a high efficiency scintillation counters are recommended to obtain a high counting rate. Also wide energy windows should be set in order to increase the count rate. A continuous energy spectrum of three $\gamma$ annihilation has to be taken into account in STOP energy window setting.

It is much more important to assure good spectrometer linearity than to improve the shape of the resolution function. Analysing the spectra obtained using the positron beam (FWHM $\sim 600 \mathrm{ps}$ ) shows that moderate misfit in the resolution function range does not influence long component significantly. The long lifetime part of the spectra is much more sensitive to the background level than the short lifetime part resulting in large distortions especially if the distribution of lifetimes is assumed.

The use of MELT for long lifetime spectra deconvolution demands an assumption of a possibly precise approximation of the resolution function and an exact time zero value. Even then it is often hard to choose a correct maximum entropy value to obtain an appropriate solution. Another disadvantage is also the MELT's tendency to split a wide distribution of lifetimes into a few narrow ones.

\section{Acknowledgments}

Author thanks J. Wawryszczuk (Maria Curie-Sklodowska University) for valuable discussions and remarks; M. Weber, K.G. Lynn and their co-workers (Washington State University) for making the positron beam spectra available; W. Volksen and R.D. Miller (IBM, Almaden Res. Centr.) for supplying the MSSQ samples and J. Goworek (Maria Curie-Skłodowska University) for supplying MCM-41 samples.

\section{References}

[1] P. Kirkegaard, M. Eldrup, Comput. Phys. Commun. 3, 240 (1972).

[2] J. Kansy, Nucl. Instrum. Methods A 374, 235 (1996). 
[3] R.B. Gregory, Nucl. Instrum. Methods A 302, 496 (1991).

[4] A. Shukla, M. Peter, L. Hoffman, Nucl. Instrum. Methods A 335, 310 (1993).

[5] R. Zaleski, J. Wawryszczuk, T. Goworek, to be published in Radiat. Phys. Chem.

[6] G. Consolati, R. Rurali, M. Stefanetti, Chem. Phys. 237, 493 (1998).

[7] G. Consolati, G. Dotelli, F. Quasso, J. Appl. Phys. 86, 4225 (1999).

[8] G. Dlubek, S. Eichler, Ch. Hübner, Ch. Nagel, Phys. Status Solidi A 174, 313 (1999).

[9] G. Dlubek, Ch. Hübner, S. Eichler, Nucl. Instrum. Methods B 142, 191 (1998).

[10] C.T. Kresge, M.E. Leonowicz, W.J. Roth, J.C. Vartuli, J.S. Beck, Nature 359, 710 (1992).

[11] T. Goworek, W. Górniak, J. Wawryszczuk, Nucl. Instrum. Methods A 321, 560 (1992).

[12] C.L. Wang, M.H. Weber, K.G. Lynn, K.P. Rodbell, Appl. Phys. Lett. 81, 4413 (2002).

[13] A. Shukla, L. Hoffmann, A.A. Manuel, M. Peter, Mater. Sci. Forum 255-257, 233 (1997). 